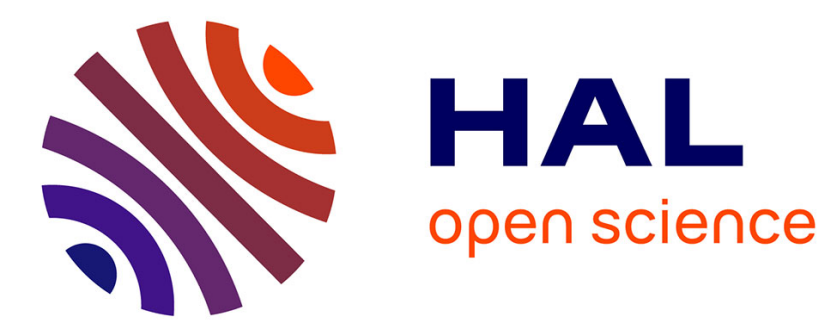

\title{
Local Registration and Deformation of a Road Cartographic Database on A SPOT Satellite Image
}

\author{
Guillaume Rellier, Xavier Descombes, Josiane Zerubia
}

\section{To cite this version:}

Guillaume Rellier, Xavier Descombes, Josiane Zerubia. Local Registration and Deformation of a Road Cartographic Database on A SPOT Satellite Image. [Research Report] RR-3939, INRIA. 2000, pp.20. inria-00072711

\section{HAL Id: inria-00072711 \\ https://hal.inria.fr/inria-00072711}

Submitted on 24 May 2006

HAL is a multi-disciplinary open access archive for the deposit and dissemination of scientific research documents, whether they are published or not. The documents may come from teaching and research institutions in France or abroad, or from public or private research centers.
L'archive ouverte pluridisciplinaire HAL, est destinée au dépôt et à la diffusion de documents scientifiques de niveau recherche, publiés ou non, émanant des établissements d'enseignement et de recherche français ou étrangers, des laboratoires publics ou privés. 
INSTITUT NATIONAL DE RECHERCHE EN INFORMATIQUE ET EN AUTOMATIQUE

\section{Local registration and deformation of a road cartographic database on a SPOT satellite image}

Guillaume Rellier — Xavier Descombes — Josiane Zerubia

$\mathbf{N}^{\circ} 3939$

Mai 2000

THÈME 3 



\title{
Local registration and deformation of a road cartographic database on a SPOT satellite image
}

\author{
Guillaume Rellier, Xavier Descombes, Josiane Zerubia \\ Thème 3 - Interaction homme-machine, \\ images, données, connaissances \\ Projet Ariana \\ Rapport de recherche $\mathrm{n}^{\circ} 3939$ - Mai 2000 - 20 pages
}

\begin{abstract}
Herein, we propose a local registration method for cartographic road networks on SPOT satellite images based on Markov Random Fields (MRF) on graphs. Since the cartographic and image data are obtained from exogeneous sources, the noises degrading these data are of different nature. This phenomenon can create important differences between the data. In addition, cartographers sometimes introduce distortions, in the so-called generalization process, in the road map in order to emphasize some details of the road (like the bends of a mountain road). The proposed algorithm aims at correcting the error due to noise and generalization, hence increasing the accuracy of the road map. The proposed method consists in translating the cartographic data into a graph model, and then defining a MRF to fit the graph on the image.
\end{abstract}

Key-words: Markov random fields, road networks, remote sensing, registration, cartography. 


\section{Recalage local et déformation d'un réseau routier cartographique sur une image SPOT}

Résumé : Dans ce rapport, nous présentons une méthode pour le recalage local d'un réseau cartographique routier sur une image SPOT, reposant sur l'utilisation des champs de Markov sur graphe. Les données image et cartographique étant obtenues par des sources exogènes, elles sont dégradées par du bruit de nature différente. Ce phénomène peut être à l'origine de différences importantes entre les données. De plus, les cartographes peuvent parfois introduire des distortions dans les cartes afin de souligner certains détails que presente la route (lacets d'une route de montagne) : c'est la généralisation. L'algorithme proposé vise à corriger les erreurs dues au bruit et à la généralisation, et à améliorer la précision du tracé des routes. La méthode proposée consiste à transformer la donnée cartographique en un graphe, et ensuite à définir un champ de Markov afin de faire correspondre le graphe et l'image

Mots-clés : Champs de Markov, réseaux routiers, télédetection, recalage, cartographie. 


\section{Contents}

1 Introduction 4

2 Modeling of the data 5

3 A markovian model for local registration and distortion of road $\begin{array}{ll}\text { maps } & 6\end{array}$

3.1 Bayesian context . . . . . . . . . . . . . . . 6

3.2 Map-dependent energy . . . . . . . . . . . . . . . . . 7

3.3 Prior energy . . . . . . . . . . . . . . . . . . . 9

$\begin{array}{llr}4 & \text { Results } & 13\end{array}$

$\begin{array}{llr}5 & \text { Conclusion } & 18\end{array}$

$\mathrm{RR} \mathrm{n}^{\circ} 3939$ 


\section{Introduction}

The aim of this work is to match, through registration and distortion, a road cartographic database on a satellite image. Thus, part of the work consists in the registration of the road map. This is a prerequisite for a further treatment since it may be hard to increase the accuracy of the details if the road is not located at a correct position. This problem is usually treated by matching linear features extracted from the image with the different pieces of the map [14] [4]. The principle of those methods is to make associations between features from each source (the map and the image), following some geometrical constraints. The difficulty of such an approach is to build a set of features from the image which is complete and does not contain false alarms. Moreover, a characteristic of this approach is that the obtained registrated graph is the result of a global rigid transformation of the given graph. This is a problem with networks presenting local errors, as it is the case with generalization. One interest of the proposed method is that it performs a local registration instead.

Another interesting aspect of the proposed algorithm consists of a network distortion in order to improve the accuracy. In other words, we want to use a road detection tool on the image so that the network better fits the image. There are many ways to compute the path joining two points supposed to be situated on the same road. A classical approach is to use gradient or Laplacian filtering, such as a Canny-Deriche filter [2] [3], possibly followed by hysteresis thresholding, and to build linear features from the result [8]. This method is fast, but it does not take into account any prior knowledge about the network shape and the connection of the network is not adressed. Another way is to use active contours, or snakes [9]. The problem of this method is that the initialization of the contour has to be close to the real road, to avoid local minima. Methods based on dynamic programming, alone [11] [6] or mixed with stochastic models [1], have also proven to be powerful. Their main drawback is the definition of starting and ending points, which requires some supervision. Herein, these points are automatically defined within the proposed algorithm.

We propose a method for registration based on a graph modeling of the road database, and the use of Markov Random Fields on this graph. In section 2, we present the modeling of objects and data, in particular the graph modeling of the road networks. In section 3 , we describe the Bayesian framework and the probabilistic model used for performing the registration and deformation. Section 4 is devoted to some registration results using the proposed method. 


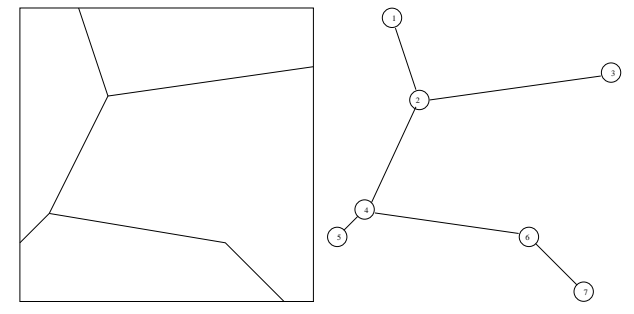

Figure 1: A cartographic network and the associated graph modeling

\section{Modeling of the data}

This particular problem of registration involves two different kinds of data, and therefore needs a previous definition of the object used for the representation. The first kind is a SPOT satellite panchromatic image, considered as a reference for registration. For the second type of data, the road map, it is not obvious at first to guess the most adapted representation for the problem.

There are many ways to represent a linear network. In the case of roads, it is possible to use pixel chains or to approximate the roads by linear primitives.

In the proposed approach, we use a non-oriented graph to model the network, $G=\{X, U, A\}, X$ being the set of nodes, $U$ the set of edges and $A$ the set of node attributes. In the proposed model, the nodes are characteristic points of the network (junctions, high curvature points), and the edges define the roads that join them. The only attribute of each node is its position in the image. These attributes are considered as random variables w.r.t. which a probabilistic model is built. The roads between the nodes are computed in a deterministic way given the nodes position. The graph is built using the information of shape (number of nodes and links between them) and position of the cartographic data. Local registration is performed by adjusting the nodes position and distortion of edges is performed by deterministic "road detection". Handling the graph is thus very simple.

This modeling is in accordance with the fact that the cartographic data are locally inaccurate and approximated, making the use of the information between nodes not reliable. It also allows to use efficient road tracking algorithms in addition to a simple registration algorithm acting on the nodes only.

Figure 1 gives an example of correspondance between a road map and its graph modeling where nodes are symbolized as circles.

$\mathrm{RR} \mathrm{n}^{\circ} 3939$ 


\section{A markovian model for local registration and distor- tion of road maps}

\subsection{Bayesian context}

The approach we propose consists in performing a deformation of the given road network in order to fit the image. Therefore we consider that the target network $X$ has a graph structure identical to the graph defined from the cartographic data. In other words, the searched network $x$ will be obtained from the initial data $m$ by moving its nodes.

This field $X$ is assumed to follow a prior probability $P(X=x)$ (noted $P(x)$ hereafter). The data are modeled as follows :

- the cartographic data $M$ is assumed to be a graph similar to $X$, i.e. a graph with the same "structure" (same number of nodes and edges), only the node attributes may differ,

- the image data $I$ is a SPOT satellite image, naturally represented as an array of pixel values.

The fields $X$ and $M$ are viewed as mutual deformation of each other, and are both assumed to be mutually dependant random variables. The image $I$ parametrizes the encountered solution, through a parametrization of the probabilities. This means that the prior, the posterior probability and the likelihood depend on the parameter $I$ and are noted respectively $P_{I}(x), P_{I}(x \mid m)$ and $P_{I}(m \mid x)$ (or resp. $P(x), P(x \mid m)$ and $P(m \mid x)$ for convenience).

The application of the Bayesian rule gives for the posterior probability of $X$ :

$$
P_{I}(x \mid m)=\frac{P_{I}(m \mid x) P_{I}(x)}{P_{I}(m)}
$$

And since the map $m$ is given :

$$
P_{I}(x \mid m) \propto P_{I}(m \mid x) P_{I}(x)
$$

Modeling the likelihood and the prior by Gibbs fields, we have $P(m \mid x)=\frac{\exp \left(-U_{m}(x)\right)}{Z_{m}}$ and $P(x)=\frac{\exp \left(-U_{a p}(x)\right)}{Z_{a p}}$

We assume that the total energy $U(x)=U_{m}(x)+U_{a p}(x)$ is a markovian energy, so that it can be written as follows : 


$$
U(x)=\sum_{c \in C} U_{c}(x)
$$

Where $C$ represents the cliques (i.e. finite sets of neighbor nodes). Taking this assumption means we assume that there exist interactions only between neighbor sites. This is a perfectly natural assumption, since if we choose for example a second order neighborhood system (neighbor nodes are those joined by an edge), each clique will represent a road section, and the energy will be a sum of terms depending on the sections.

The solution is the configuration which optimizes the posterior in the sense of a Bayesian criterion. We consider the maximum a posteriori (MAP) estimator defined by :

$$
\hat{x}=\arg \min _{x}\left(U_{m}(x)+U_{a p}(x)\right)
$$

To reach the solution, we use simulated annealing which assures convergence to the global minimum [7].

We now define the two components of the proposed model total energy : $U(x)=$ $U_{m}(x)+U_{a p}(x)$.

\subsection{Map-dependent energy}

The map-dependent term of the energy, $U_{m}(x)$, expresses the consistency between the graph $x$ and the data $m$. Roughly speaking, a configuration of $x$ has a low energy if each road section of $x$ is similar to its equivalent in $m$, and if the connections between sections are also similar. This yields two types of constraints :

- the first constraint operates on pairs of nodes connected by a section of road. It depends on the distance between the two nodes, which must be close to the corresponding distance in the map,

- the second constraint operates on series of three nodes joined by two sections. It depends on the angle formed by the nodes, which again must be close to the corresponding angle in the map.

From these two constraints, it is possible to build the map-dependent energy function in the following way.

Define a graph-associated distance :

$\mathrm{RR} \mathrm{n}^{\circ} 3939$ 


\section{DEFINITION 1}

A path between $x_{i}$ and $x_{j}$ is a set of nodes $\left(x_{i}=x_{0}, x_{1}, \ldots, x_{N}=x_{j}\right)$ where two consecutive nodes $x_{k}$ and $x_{k+1}$ are joined by an edge.

DeFinition 2 (GRAPH Distance)

The distance between two points $x_{i}$ and $x_{j}$ of a graph $X$, noted $d\left(x_{i}, x_{j}\right)$, is equal to the minimum number of edges for every path $\left(x_{i}, x_{j}\right)$ (which is equal to the number of nodes minus one).

For example, the distance between to nodes joined by an edge is 1 .

We define the map-dependent energy as :

$$
U_{m}(x)=\sum_{d\left(x_{i}, x_{j}\right)=1} U_{m}^{1}\left(x_{i}, x_{j}\right)+\sum_{\begin{array}{c}
d\left(x_{i}, x_{j}\right)=1 \\
d\left(x_{j}, x_{k}\right)=1
\end{array}} U_{m}^{2}\left(x_{i}, x_{j}, x_{k}\right)
$$

This is a Markovian energy w.r.t. the following neighborhood system :

$$
x_{j} \in V_{x_{i}} \text { if and only if }\left\{\begin{array}{l}
x_{i} \neq x_{j} \\
d\left(x_{i}, x_{j}\right) \leq 2
\end{array}\right.
$$

The potentials $U_{m}^{1}\left(x_{i}, x_{j}\right)$ and $U_{m}^{2}\left(x_{i}, x_{j}, x_{k}\right)$ stand respectively for the first and the second constraints defined previously.

$$
U_{m}^{1}\left(x_{i}, x_{j}\right)=f_{i j}\left(\operatorname{dist}\left(x_{i}, x_{j}\right)\right)
$$

where $\operatorname{dist}(a, b)$ is the Euclidean distance between node $a$ and $b$.

And :

$$
U_{m}^{2}\left(x_{i}, x_{j}, x_{k}\right)=g_{i, j, k}\left(\alpha_{i, j, k}\right)
$$

where $\alpha_{i, j, k}$ is the angle formed by the two segments $\left[x_{i}, x_{j}\right]$ and $\left[x_{j}, x_{k}\right]$.

The function $f_{i j}$, whose argument is the Euclidean distance between two nodes is a convex function taking its minimum value when $\operatorname{dist}\left(x_{i}, x_{j}\right)=\operatorname{dist}\left(x_{i}^{m}, x_{j}^{m}\right)$ (where $\operatorname{dist}\left(x_{i}^{m}, x_{j}^{m}\right)$ is the Euclidean distance of the corresponding nodes in the map).

The function $g_{i, j, k}$ takes as argument the value of the angle formed by the considered three consecutive nodes. Its characteristics is to be defined for the interval $[0,2 \pi]$, to take its minimum for $\alpha_{i, j, k}=\alpha_{i, j, k}^{m}$ (where $\alpha_{i, j, k}^{m}$ is the angle of the corresponding nodes in the map), and to increase with the difference between $\alpha_{i, j, k}$ and $\alpha_{i, j, k}^{m}$. 
For the experiments, $f_{i j}$ and $g_{i, j, k}$ were defined as follows :

$$
\begin{gathered}
f_{i j}(d)=\left(d-\operatorname{dist}\left(x_{i}^{m}, x_{j}^{m}\right)\right)^{2} \\
g_{i j k}(\alpha)=\left\{\left(\alpha-\alpha_{i, j, k}^{m}\right)[2 \pi]\right\}^{2}
\end{gathered}
$$

Therefore, the optimal configurations with respect to this first term are the configuration equal to the map and the configurations obtained by any rigid transformation of the data. Configurations consisting of small local deformations of these are also close to optimality.

\subsection{Prior energy}

The term $U_{a p}(x)$ takes into account the information given by the image, since this energy depends on the parameter $I$. It is composed of two terms, both depending on the parameter $I$, directly or indirectly.

The treatment of the edges of the graph $x$ is the starting point of prior energy definition. The edges represent the road sections joining the different nodes of the network. They are represented as segments on the map. Therefore, they have to be distorted to match the SPOT satellite image. The chosen solution is to compute the expected section of the road between two nodes in a deterministic way using a dynamic programming algorithm. The resulting path between two nodes only depends on their position in the image and the grey level configuration of the image.

The path between two connected nodes is obtained by minimizing a cost function (depending on the contrast between the path and the background, the gray level along the path and the local curvature). The minimization of this cost function is performed by the dynamic programming algorithm presented in [11] [10], derived from the $\mathrm{F}^{*}$ algorithm [5], thanks to the ELIESER program, implementing this algorithm.

Let $x_{i}$ and $x_{j}$ be the two nodes ending the considered edge. Define a function $\Phi$, called potential. This function is defined on the set of three distinct pixel series $s_{i}, s_{j}$ and $s_{k}$, such that $s_{i}$ and $s_{j}$ on one hand, and $s_{j}$ and $s_{k}$ on the other, are neighbor in 8-connectivity ; $s_{i}$ and $s_{k}$ must not be neighbors, in order to limit the local curvature. This yields three different places for $s_{k}$ when $s_{i}$ and $s_{j}$ are fixed (see figure 2).

The potential $\Phi$, or cost function, is designed in order to penalize pixel series which do not match road characteristics. Pixel series with gray level configuration matching the following constraints will have a low cost :

$\mathrm{RR} \mathrm{n}^{\circ} 3939$ 


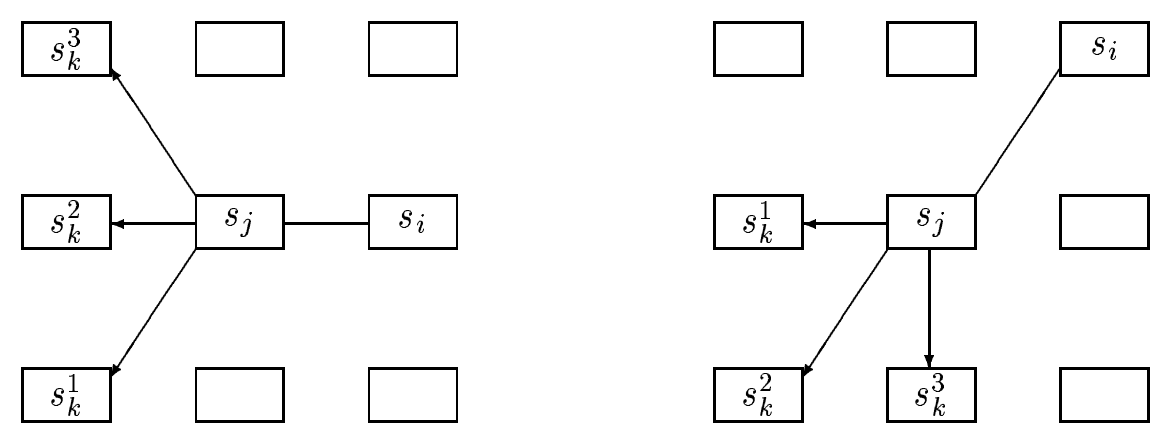

Figure 2: The possible $s_{k}$ positions, $s_{i}$ and $s_{j}$ being fixed.

- gray level homogeneity along the series,

- contrast between the three road pixels and the six neighbors.

We also add a local curvature constraint, penalizing curved paths in favor of straight paths. This yields the following expression :

$$
\begin{gathered}
\Phi=\Phi_{1}+\Phi_{2}+\Phi_{3} \\
\Phi_{1}\left(x_{i}, x_{j}, x_{k}\right)=\alpha\left(\max \left(x_{i}, x_{j}, x_{k}\right)-\min \left(x_{i}, x_{j}, x_{k}\right)\right) \\
\Phi_{2}\left(x_{i}, x_{j}, x_{k}\right)=\beta\left\|x_{m e d 1}-x_{m e d 2}\right\|
\end{gathered}
$$

Where $x_{m e d 1}$ and $x_{m e d 2}$ are respectiveley the median value of the three road pixels and the median value of the six background pixels.

$$
\Phi_{3}\left(x_{i}, x_{j}, x_{k}\right)=\left\{\begin{array}{l}
0 \text { if the three pixels are on a straight line } \\
\gamma \text { if the three pixels exhibit a } \pi / 4 \text { angle }
\end{array}\right.
$$

Let a path $p$ be defined by the series of pixels $s_{0}=x_{i}, \ldots, s_{k}, s_{k+1}, \ldots, s_{N}=x_{j}$ such that each three consecutive pixel series belongs to the domain of definition of $\Phi$. Define the energy of the path $p$ :

$$
U(p)=\sum_{x_{i}}^{x_{j}} \Phi\left(s_{i-1}, s_{i}, s_{i+1}\right)
$$

Because of the definition of the cost function $U$, the target path $\hat{p}$ is such that : 

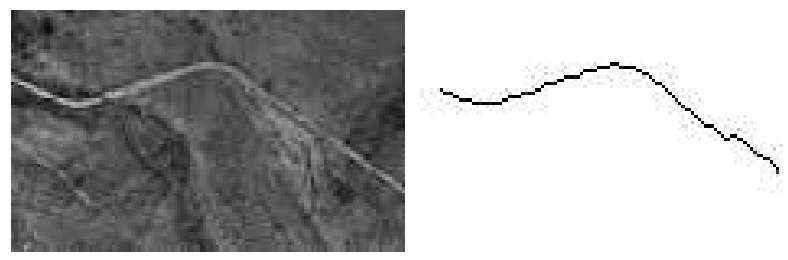

Figure 3: right, the road joining two extremities given by ELIESER ; left, the corresponding piece of SPOT image.

$$
\hat{p}=\arg \min _{p} U(p)=\min _{p} \sum_{x_{i}}^{x_{j}} \Phi\left(s_{i-1}, s_{i}, s_{i+1}\right)
$$

Then the problem reduces to find this minimum energy path. The minimal path is obtained using a dynamic programming scheme. The algorithm is based on $\mathrm{F}^{*}$, and consists in sweeping the image in four directions and performing a summationminimization process, using potential $\Phi$ (see [11] for more details).

As a result of this step we obtain, for a given configuration of $x$, the expected paths between neighboring nodes which will be used to compute the prior energy.

The prior energy is depends if the computed network, given the node configuration $x$, is likely to be a real road network or not. Two points of view are taken into account in the prior energy. The first one defines some geometrical aspect, and the second one is about compatibility with the image data. This yields the expression for prior energy :

$$
U_{a p}(x)=U_{a p, 1}(x)+U_{a p, 2}(x)
$$

The first term expresses geometrical constraints on the network. This term aims at avoiding that two sections joining two separated pairs of nodes merge even partially, since by the definition of the graph, each couple of nodes represents a separate section.

Some roads can be very pronounced, and thus attract unexpected pairs of nodes, because of the very low image-dependent potential they provide. This is the reason why this "non-overlapping" energy $U_{a p, 1}(x)$ is needed. The potential associated to $U_{a p, 1}(x)$ is equal to the number of pixels the considered section of road has in common with the other sections of road.

The expression of $U_{a p, 1}(x)$ is given by : 


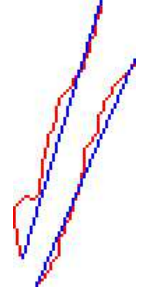

a)

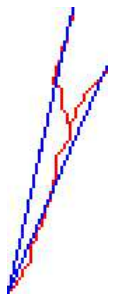

b)

Figure 4: Two possible configurations for two pairs of nodes : influence of the node positions on the computed paths (in red) which can overlap if some nodes are too near (right)

$$
U_{a p, 1}(x)=\sum_{s_{1}, s_{2} \in S} U_{a p, 1}^{s_{1}, s_{2}}(x)=\sum_{\begin{array}{l}
d\left(x_{i}, x_{j}\right)=1 \\
d\left(x_{k}, x_{l}\right)=1
\end{array}} U_{a p, 1}\left(x_{i}, x_{j}, x_{k}, x_{l}\right)
$$

where $s_{1}=\left(x_{i}, x_{j}\right)$ is the road section joining $x_{i}$ and $x_{j}, s_{2}=\left(x_{k}, x_{l}\right)$ the road section joining $x_{k}$ and $x_{l}$, and the potential is :

$$
U_{a p, 1}^{s_{i}, s_{j}}(x)=n_{i j}
$$

where $n_{i j}$ is the number of common pixels between $s_{i}$ and $s_{j}$.

Figure 4 shows a case where the first term will penalize the fact that two roads overlap (on the right hand side), and will favour a configuration with no overlapping, like the left one.

The second part of the prior energy $U_{a p}^{2}(x)$ is directly related to the image. It gives a measure, for the computed path of each couple of nodes, related to how the grey level configuration characteristics of the path in the image are road-like characteristics.

Choosing an intuitive neighborhood system in which neighbor nodes are those joined by a section of road, we have :

$$
U_{a p, 2}(x)=\sum_{d_{i j}=1} U_{a p, 2}\left(x_{i}, x_{j}\right)=\sum_{s \in S} U_{a p, 2}^{s}(x)
$$

where $S$ is the set of road sections $s$ and $d_{i j}$ the graph-distance between two nodes $x_{i}$ and $x_{j}$. 
Each term $U_{a p, 2}^{s}(x)$ expresses the probability for the considered section $s$ to be situated at the current position given the image data.

A road is defined as a linear structure forming exhibiting a contrast with respect to the background. We thus define three zones : a "road" zone, a "background" zone, and an "intermediate" zone (to deal with different road thicknesses), as shown in figure 5. We calculate the means of the "road" and the "background" zones. Pixels of the intermediate zone are classified as "road" (resp. "background") if their value is closer to the "road" (resp. "background") mean than to the "background" (resp. "road") mean.

A statistical hypothesis test between the obtained zones is then computed. This test discriminates between the two gray level distributions. We choose the student $t_{-}$test :

$$
t_{-} \text {test }\left(D_{1}, D_{2}\right)=\frac{\mu_{1}-\mu_{2}}{\sqrt{\left(\frac{N_{1} \sigma_{1}^{2}+N_{2} \sigma_{2}^{2}}{N_{1}+N_{2}-2}\right)\left(\frac{1}{N_{1}}+\frac{1}{N_{2}}\right)}}
$$

A decreasing function of the test value gives the image-dependent potential of the considered section of road :

$$
U_{a p, 2}^{s}(x)=\Psi\left(t_{-} \operatorname{test}\left(D_{1}, D_{2}\right)\right)
$$

where $D_{1}$ and $D_{2}$ are the "background" and the "road" grey level distributions along path computed for section $s, \Psi(x)=\lambda / x$ and $\lambda$ is a parameter to be set. Using this test for the prior energy is more accurate than using directly the energy given by ELIESER, because the latter strongly depends on the number of pixels along the path. For example, if ELIESER potential is positive for all series of three pixels, this energy would have a tendency to prefer short roads, and even roads reduced to a point.

Finally, the expression of the prior energy is :

$$
U_{a p}(x)=\sum_{s_{i}, s_{j} \in S} U_{a p, 1}^{s_{i}, s_{j}}(x)+\sum_{s \in S} U_{a p, 2}^{s}(x)
$$

where $S$ is the set of road sections, that is the set of couples of nodes joined by an edge.

\section{Results}

To optimize the proposed model we run a simulated annealing algorithm [13].

$\mathrm{RR} \mathrm{n}^{\circ} 3939$ 


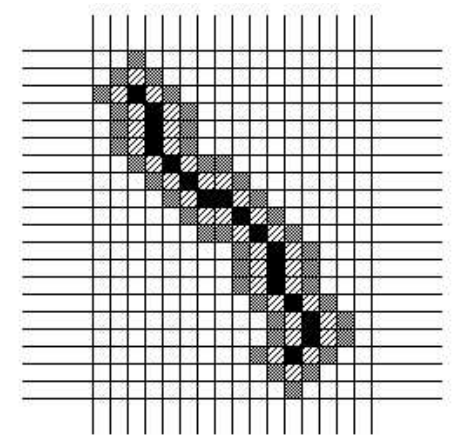

Figure 5: The 3 different zones for the test : the road (in black), the background (in grey), the intermediate zone (hatched).

Herein, we sample the field $x$ using a Metropolis dynamics [12]. For each node successively, we draw a new position within a window centered on this node position, and validate or not using Metropolis dynamics :

1. Initialization : $T=T_{0}, x^{(0)}$ defined by the map, $n=0$ and set the total number of iterations to $N I T$,

2. For each node $x_{i}^{(n)}$ :

2.1. Choose randomly a new position $y$ of $x_{i}$ within a window centered on the current position $x_{i}^{(n)}$,

2.2. Compute the conditional energy variation of the movement $x_{i}^{(n)}->y$

$$
\Delta U=U\left(y \mid N_{i}\right)-U\left(x_{i}^{(n)} \mid N_{i}\right)
$$

where $N_{i}$ is $x_{i}$ 's neighborhood,

2.3. If $\Delta U \leq 0$, then $x_{i}^{(n)}=y$,

If $\Delta U>0$, then $x_{i}^{(n)}=y$ with probability $p=\exp (-\Delta U)$,

3. If $n=N I T$ stop, else $n=n+1$ and $T^{(n+1)}=\frac{\log (n+1)}{\log (n+2)} T^{(n)}$,

4. Goto 2. 


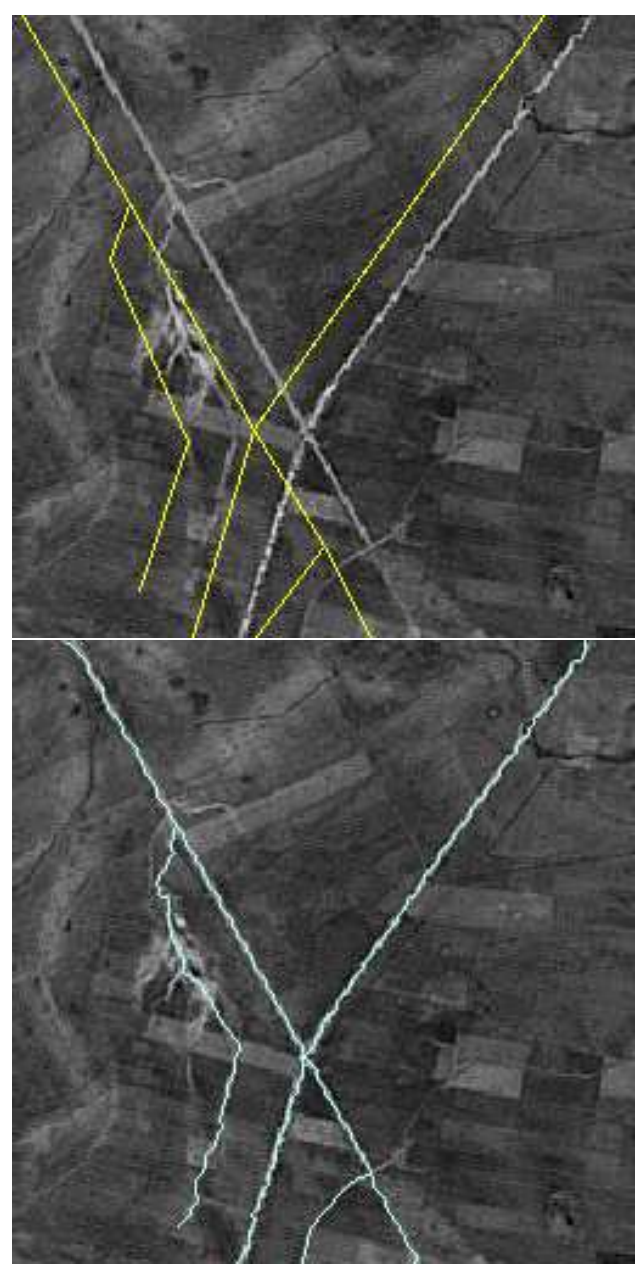

Figure 6: Example of result (bottom) obtained from a shifted map (top)

Experiments are performed on SPOT satellite images using cartographic data artificially corrupted with noise, i.e. artificially shifted, rotated or deformed.

Figure 6 shows the result with an initial map shifted by 15 pixels from the real cartographic data. The result is the expected one, despite an important initial shift.

Figure 7 shows the result of the algorithm with an initial map rotated by 0.2 radian from the real cartographic data. The nodes have been located at the right 


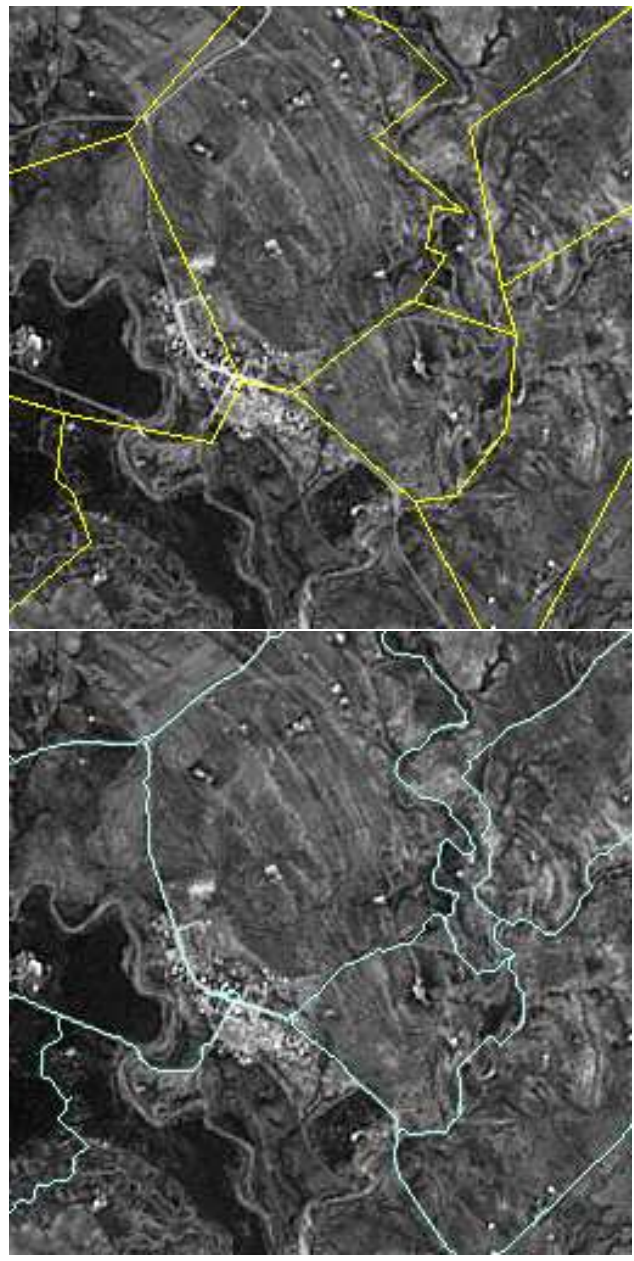

Figure 7: Example of result (bottom) obtained from a rotated map (top)

place. Small errors appear in the upper right part of the image due to a very low SNR.

Finally we introduce noise on the distances and on the angles by randomly translating each node of the initial graph within an 10 by 10 pixel window centered on the exact position. 


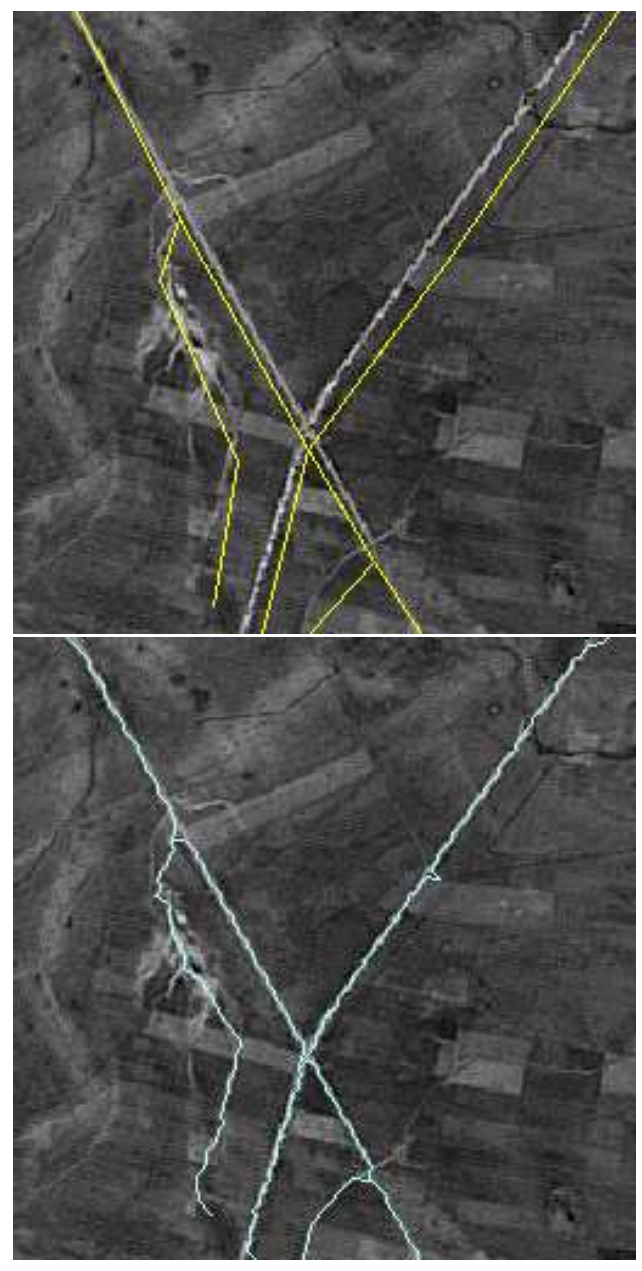

Figure 8: Example of result (bottom) obtained from a distorted map (top) 


\section{Conclusion}

Herein, we have proposed a model to locally register a map on a satellite image and to improve maps accuracy. This model, based on Markov Random Fields, gives acceptable results for noisy images or road maps. The model is robust towards global position errors of the map, for instance when there is a shift or a rotation. One interesting point of the model is that the registration is done locally, and not through a global rigid transformation. This is very useful when the map exhibits local errors, for example due to generalization. Another point of interest is the coupling of stochastic and deterministic methods, since completely stochastic methods (with stochastic road detection) would have a prohibitive computational cost, and completely deterministic methods would be very sensitive to local minima and initialization.

An open problem is still the computational time, which remains long because of the frequent computations of the path between nodes. Also a further investigation could be the addition, and perhaps the removal of roads, for applications like updating cartographic databases. 


\section{References}

[1] Meir Barzohar and David B. Cooper. Automatic finding of main roads in aerial images by using geometric-stochastic models and estimation. IEEE Trans. on Pattern Analysis and Machine Intelligence, 18(7):707-721, July 1996.

[2] John Canny. A computational approach to edge detection. IEEE Trans. on Pattern Analysis and Machine Intelligence, 8(6):679-698, 1986.

[3] Rachid Deriche. Using Canny's criteria to derive a recursively implemented optimal edge detector. International Journal of Computer Vision, 1(2):167187, 1987.

[4] Xavier Descombes, Christine Hivernat, Sandrine Randriamasy, and Josiane Zerubia. Graph-matching model using Gibbsian modeling : application to mapspot image road networks for map updating. In Proc. SPIE, pages 2-10, Denver, July 1999.

[5] M.A. Fischler, J.N. Tennenbaum, and H.C. Wolf. Detection of roads and linear structures in low-resolution aerial imagery using a muiltisource knowledge integration technique. CVGIP, 15:201-223, 1981.

[6] Davi Geiger, Alok Gupta, Luiz A. Costa, and John Vlontzos. Dynamic programming for detecting, traking and matching deformable contours. IEEE Trans. on Pattern Analysis and Machine Intelligence, 17(3):294-302, March 1995.

[7] Stuart Geman and Donald Geman. Stochastic relaxation, Gibbs distribution and the Bayesian restauration of images. IEEE Trans. on Pattern Analysis and Machine Intelligence, 6(6):721-741, November 1984.

[8] Gerard Giraudon. Chaînage efficace de contour. INRIA Research Report (in French) 605, 1987.

[9] Michael Kass, Andrew Witkin, and Demetri Terzopoulos. Snakes : Active contour models. International Journal of Computer Vision, 1(4):321-331, 1988.

[10] Nicolas Merlet and Josiane Zerubia. A curvature-dependent energy function for detecting lines in satellite images. In Proc. 8th SCIA, May 1993.

[11] Nicolas Merlet and Josiane Zerubia. New prospects in line detection by dynamic programming. IEEE Trans. on Pattern Analysis and Machine Intelligence, 18(4):426-431, April 1996.

$\mathrm{RR} \mathrm{n}^{\circ} 3939$ 
[12] N Metropolis, A.W. Rosenbluth, N.M. Rosenbluth, A.H. Teller, and E. Teller. Equation of state calculations by fast computing machines. J. Chemical Physics, 21:1087-1091, 1953.

[13] Peter J.M. van Laarhoven and Emile H.L. Aarts. Simulated annealing : theory and applications. D. Reidel, 1987.

[14] Richard C. Wilson and Edwin R. Hancock. Structural matching by discrete relaxation. IEEE Trans. on Pattern Analysis and Machine Intelligence, 19(6):634648, June 1997. 


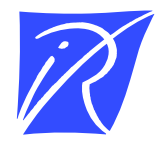

Unité de recherche INRIA Sophia Antipolis 2004, route des Lucioles - B.P. 93 - 06902 Sophia Antipolis Cedex (France)

Unité de recherche INRIA Lorraine : Technopôle de Nancy-Brabois - Campus scientifique 615, rue du Jardin Botanique - B.P. 101 - 54602 Villers lès Nancy Cedex (France)

Unité de recherche INRIA Rennes : IRISA, Campus universitaire de Beaulieu - 35042 Rennes Cedex (France)

Unité de recherche INRIA Rhône-Alpes : 655, avenue de l'Europe - 38330 Montbonnot St Martin (France)

Unité de recherche INRIA Rocquencourt : Domaine de Voluceau - Rocquencourt - B.P. 105 - 78153 Le Chesnay Cedex (France)

Éditeur

INRIA - Domaine de Voluceau - Rocquencourt, B.P. 105 - 78153 Le Chesnay Cedex (France)

http://www.inria.fr

ISSN 0249-6399 